\title{
Time evolution of X-ray coronal activity in PMS stars; a possible relation with the evolution of accretion disks ${ }^{\star}$
}

\author{
E. Flaccomio, G. Micela, and S. Sciortino
}

\author{
INAF - Osservatorio Astronomico di Palermo Giuseppe S. Vaiana, Palazzo dei Normanni, 90134 Palermo, Italy \\ e-mail: giusi@oapa.astropa.unipa.it, sciorti@oapa.astropa.unipa.it
}

Received 24 September 2002 / Accepted 6 February 2003

\begin{abstract}
We investigate the evolution of X-ray stellar activity from the age of the youngest known star forming regions (SFR), $\lesssim 1 \mathrm{Myr}$, to about $100 \mathrm{Myr}$, i.e. the zero age main sequence (ZAMS) for a $\sim 1 M_{\odot}$ star. We consider five SFR of varying age ( $\rho$ Ophiuchi, the Orion Nebula Cluster, NGC 2264, Chamaeleon I, and $\eta$ Chamaeleontis) and two young clusters (the Pleiades and NGC 2516). Optical and X-ray data for these regions are retrieved both from archival observations and recent literature, and reanalyzed here in a consistent manner so as to minimize systematic differences in the results.

We study trends of $L_{\mathrm{X}}$ and $L_{\mathrm{X}} / L_{\mathrm{bol}}$ as a function of stellar mass and association age. For low mass stars $\left(M \lesssim 1 M_{\odot}\right)$ we observe an increase in $L_{\mathrm{X}} / L_{\mathrm{bol}}$ in the first 3-4 Myr and a subsequent leveling off at the saturation level $\left(L_{\mathrm{X}} / L_{\mathrm{bol}} \sim-3\right)$. Slowly evolving very low mass stars then retain saturated levels down to the oldest ages here considered, while for higher mass stars activity begins to decline at some age after $\sim 10^{7}$ years.

We find our data consistent with the following tentative picture: low mass PMS stars with no circumstellar accretion disk have saturated activity, consistently with the activity-Rossby number relation derived for MS stars. Accretion and/or the presence of disks somehow lowers the observed activity levels; disk dissipation and/or the decrease of mass accretion rate in the first few Myrs of PMS evolution is therefore responsible for the observed increase of $L_{X} / L_{\mathrm{bol}}$ with time.
\end{abstract}

Key words. stars: activity - stars: pre-main sequence - open clusters and associations: individual: $\rho$ Ophiuchi, Orion Nebula Cluster, Chamaeleon I, $\eta$ Chamaeleontis

\section{Introduction}

Since the advent of sensitive X-ray imaging observations, late type $(>\mathrm{F})$, low mass stars are known to be sources of $\mathrm{X}$-rays with luminosities spanning more than 4 orders of magnitude, from $L_{\mathrm{X}}=10^{27}$ or less to more than $10^{31} \mathrm{ergs} / \mathrm{s}$ (Vaiana et al. 1981). Given the similarity with the solar emission (e.g. thermal emission-line X-ray spectrum, flares) it is believed that $\mathrm{X}$-ray emission from low mass stars originates from stellar coronae that are essentially similar to the solar one $\left(L_{\mathrm{X}, \odot}=0.2-\right.$ $5.0 \times 10^{27} \mathrm{ergs} / \mathrm{s}$, Peres et al. 2000), albeit sometimes much more active.

In order to observationally investigate the physical origin of the large observed spread in $L_{\mathrm{X}}$, homogeneous stellar samples have been successfully employed to restrict the number of free parameters. Open clusters and Star Forming Regions (SFR) are ideal targets in this respect, as all stars have approximatively the same age and metallicity. The comparison between different regions then ideally permits to investigate the age evolution and the metallicity dependence of X-ray activity.

\footnotetext{
Send offprint requests to: E. Flaccomio,

e-mail: ettoref@oapa.astropa.unipa.it

$\star$ Table 1 is only available in electronic form at http://www. edpsciences.org
}

For late-type slowly rotating stars of nearby field population, coronal and chromospheric activity indicators correlate with stellar rotation (Pallavicini et al. 1981) and with convective zone properties (Noyes et al. 1984; Pizzolato et al. 2003 and references therein). Such correlations however do not hold for fast rotating stars, whose observed activity levels appear to have reached saturation (e.g. $L_{\mathrm{X}} / L_{\mathrm{bol}} \sim 10^{-3}$ ). X-ray activity has also been found to decrease with increasing stellar age as stars loose angular momentum through an activity driven magnetized wind. Such observed relations are the main evidence that activity in the main sequence (MS) is produced by a stellar dynamo whose efficiency depends, at least for slow rotations, on stellar rotation and convection (the so called $\alpha-\omega$ dynamo).

While MS activity can be convincingly ascribed to the stellar dynamo, the mechanism responsible for coronal activity in low mass, pre-main-sequence (PMS) stars is not understood. During the last 20 years several close-by SFRs have been observed in the X-rays (e.g. Feigelson et al. 1993; Damiani \& Micela 1995; Flaccomio et al. 2003a,b), finding bright X-ray emission from most PMS stars. No conclusive evidence for the action of a MS-type dynamo mechanism has been found for these stars. Although the presence of a convective layer does seem to be important for the existence of an active corona (massive fully radiative PMS stars do not show coronal emission), 
no relation has been generally found between the X-ray emission level of PMS stars and their rotation periods ${ }^{1}$ and/or convective region depth (or turn-over times). Instead, a relation has been often found with stellar mass or bolometric luminosity (e.g. Feigelson et al. 1993, 2002; Flaccomio et al. 2003b), parameters unrelated to the $\alpha-\omega$ dynamo. It is therefore not clear at present whether a new mechanism should be invoked to explain coronal activity in the PMS (e.g. a different dynamo), or the same dynamo acting on the MS works here in a saturation regime, and/or additional phenomena occur in the PMS coronae that hide the underlying correlations with the $\alpha-\omega$ dynamo parameters.

The picture is probably further complicated by matter accretion phenomena occurring on many PMS stars through a circumstellar disk: either accretion or the presence of the disk may play a role in determining the observed X-ray activity levels. Several studies have indeed found that accreting stars have lower mean X-ray activity levels, and more time-variable emission respect to their non-accreting counterparts (Neuhäuser et al. 1995; Flaccomio et al. 2000; Flaccomio et al. 2003b,c; Stelzer \& Neuhäuser 2001). However, this point is controversial: other authors have failed to detect a significant difference in activity levels of accreting and non accreting stars (e.g. Feigelson et al. 2002; Preibisch \& Zinnecker 2002; Feigelson et al. 1993). As discussed by Flaccomio et al. (2003c), we believe that the discrepancy in the results is due, rather than to differences in the data themselves, to the different sensitivity of the techniques used to compare the activity levels of the two classes. In this work we will therefore assume that accretion disks do influence activity. The physical nature of such influence is presently not understood. Some hypotheses, proposed by Damiani \& Micela (1995) and Flaccomio et al. (2003b), speculate that accretion (and/or the associated outflow) may modify the geometry of the magnetic field that confines the coronal plasma, either reducing the fraction of the stellar surface available for closed magnetic loops, or modifying more radically the magnetic field, maybe with the involvement of the magnetized inner parts of the circumstellar disk (e.g. Montmerle et al. 2000).

While the study of individual SFRs and open clusters allows us to study the correlation (or lack of correlation) of X-ray activity with parameters such as stellar rotation, mass and circumstellar accretion indicators, important clues to the mechanism responsible for activity are expected to come from the comparison of SFR at different evolutionary stages.

In this work we assume, to first approximation, that the differences in activity between stars belonging to different associations can be attributed exclusively to their different evolutionary stages. In other words, we assume that, in different associations, stars of a given mass are statistically indistinguishable at the time of their formation (at least in regard to

\footnotetext{
1 An exception is the Taurus region (e.g. Stelzer \& Neuhäuser 2001). However it is also possible that the correlation between activity and rotation is in this case the consequence of the possibly more fundamental correlations between activity and mass-accretion (Flaccomio et al. 2003b,c) and between rotation and accretion (e.g. Bouvier et al. 1997).
}

activity) and that their evolution is not influenced by different ambient conditions. These assumptions may be questioned because, for example: differences in initial angular momentum and stellar metallicity may affect X-ray activity; differences in environment, such as stellar density, can affect properties of the stellar population, such as the frequency of multiple systems and the circumstellar disk lifetime (Haisch et al. 2001b) which may both influence observed X-ray activity levels. Here, neglecting these possible complications, we will directly compare results obtained for stellar associations of varying age, in an attempt to investigate the effect of stellar evolution on activity. More precisely, we will select stars in definite mass ranges and we will follow the evolution with time of two activity indicators, $L_{\mathrm{X}}$ and $L_{\mathrm{X}} / L_{\mathrm{bol}}$.

In order to complete our program, we consider here six young PMS stellar groups with ages ranging from $\sim 1$ to $\sim 7$ Myrs: $\rho$ Ophiuchi, the Orion Nebula Cluster (ONC), split in the central and outer regions (see below), NGC 2264, the Chamaeleon I association and $\eta$ Chamaeleontis. In addition we consider two older ZAMS clusters: the Pleiades and NGC 2516. These regions have been selected so to probe a significant age range and because optical and X-ray data suitable for our analysis were available either in the literature or in data archives. In all cases we try to consider stellar samples that are likely free from X-ray selection effects and, when needed, we re-analyze the published data in an homogeneous way. The latter is a crucial step because different instrumentation, assumptions and analysis techniques employed to derive physical quantities from both X-ray and optical observations can result in significant systematic differences that would interfere with our purpose.

In the following we first introduce the procedures employed to consistently analyze (or re-analyze) the data for the various regions (Sect. 2); we then discuss each of them individually, the sources of our data, the analysis performed, and the results obtained in regard to the dependence of activity on stellar mass (Sect. 3). Finally we present our evolutionary picture (Sect. 4) and propose a simple physical model that tries to explain our observational findings (Sect. 5).

\section{Generals of data analysis}

The quantities we will deal with are two X-ray activity indicators $\left(L_{\mathrm{X}}\right.$ and $\left.L_{\mathrm{X}} / L_{\mathrm{bol}}\right)$, stellar masses and ages. The estimates of these quantities are often subject to significant uncertainties, due both to errors in the observational quantities and to the assumptions made in order to convert these latter into physical parameters. In order to make the comparisons of activity among different associations meaningful, special care must be therefore taken on the one hand to reduce random uncertainties and on the other to minimize systematic differences due to the non-uniform approaches and assumptions made in the different studies. In the following two subsections we review the steps taken to this effect. 


\subsection{X-ray luminosities}

We utilize data obtained with four X-ray detectors: ROSAT HRI and PSPC, Chandra HRC-I and ACIS-I. In the original works from which these data are taken, X-ray luminosities were calculated from measured instrumental count-rates assuming different spectral bands, the choice depending largely on the spectral response of the instrument. These X-ray luminosities are not directly comparable. The count-rate to luminosity conversion factors were computed in all cases assuming optically thin isothermal plasma emission. The assumed plasma temperatures $(k T)$, however, varied from case to case, as well as the way in which interstellar extinction was accounted for. Here we recompute all conversion factors, using PIMMS $^{2}$, for a single spectral band (0.1-4.0 keV). For PMS stars we assume $k T=2.16 \mathrm{keV}$, consistent with recent results on several SFRs (e.g. Feigelson et al. 2002; Flaccomio et al. 2003a; Imanishi et al. 2001a), and an hydrogen column density, $N_{\mathrm{H}}$, proportional to optical/IR extinction, $A_{V}$ or $A_{J}$, where the latter is measured, with the exception of $\eta$ Chamaeleontis (Sect. 3.5), individually for each member of the association.

For the two MS clusters, NGC 2516 and the Pleiades, we assume (cf. Gagné et al. 1995b) lower temperatures, between $\sim 0.4$ and $\sim 1.0 \mathrm{keV}$. Given the small and quite uniform interstellar extinction, $N_{\mathrm{H}}$ was assumed, for stars lacking individual measurements, to correspond to the mean $A_{V}$ of the entire cluster.

Departure from our assumptions for $k T$ and $N_{\mathrm{H}}$ will result in errors in the count rate to $L_{\mathrm{X}}$ conversion whose magnitudes depend on the detector used for the observation and on the range of variation of the two parameters for the given stellar population. In the following we will discuss, for some of the regions considered, the sensitivity of the conversion factor to these assumptions. In all cases the likely systematic uncertainty in $L_{\mathrm{X}}$ is smaller than $\sim 0.1 \mathrm{dex}$.

\subsection{Masses and ages}

We make use of Siess et al. (2000, SDF hereafter) evolutionary tracks ${ }^{3}$ in order to estimates masses and ages of PMS stars from effective temperatures and bolometric luminosities. These latter values are taken from published works, derived in most cases from medium resolution optical or IR spectra. For the two oldest PMS regions, Chamaeleon I and $\eta$ Chamaeleontis, estimates are based on optical/IR photometry. Ages for the two ZAMS clusters are taken from Meynet et al. (1993) and are derived from the turnover point in the upper main sequence; we then estimate stellar masses from optical photometry and the theoretical isochrones appropriate to the clusters age (SDF; solar metallicity), transformed from the theoretical $L_{\mathrm{bol}}-$ $T_{\text {eff }}$ plane into the observational $V-(B-V)$ plane with the relations given by Kenyon \& Hartmann (1995). Note that masses for both PMS and MS stars are estimated from the same theoretical evolutionary model. In order to alleviate the effect of

\footnotetext{
${ }^{2}$ Portable Interactive Multi-Mission Simulator.

3 See also the web site:

http://www-laog.obs.ujf-grenoble.fr/activites/starevol/ evol.html
}

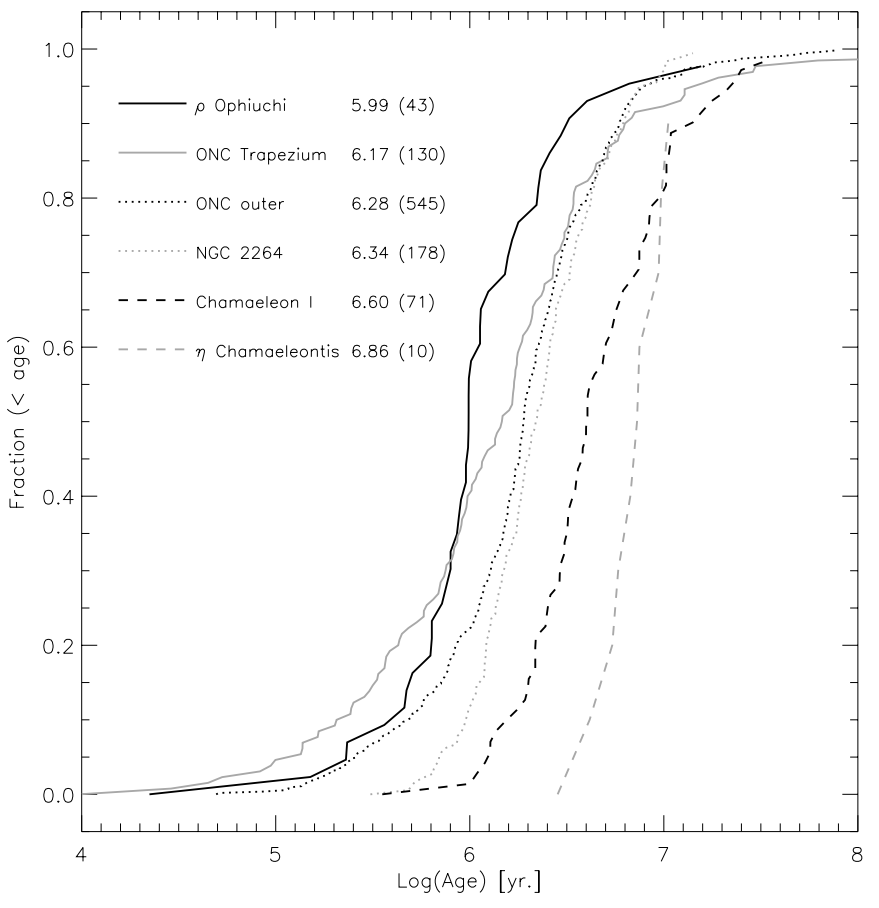

Fig. 1. Cumulative age distribution for the six PMS regions under study. The legend on the upper-left side reports, for each region, the median of the logarithm of the age (in years) and, in parenthesis the number of stars in the sample.

residual uncertainties in the mass estimates (e.g. due to the different temperature scales used in the original studies the data are taken from) we will consider wide mass ranges, similarly to what done for the ONC by Flaccomio et al. (2003b).

We now turn to the problem of PMS stellar ages: individual ages inferred from PMS evolutionary models are known to be subject to large uncertainties. In addition to the uncertainties in the theoretical evolutionary models, bolometric luminosities are subject to an artificial spread due to a number of effects (Hartmann 2001). As a result the real dispersion of stellar ages in a given star forming region may be much smaller than estimated from its HR diagram. We decided to characterize each PMS stellar group with a single age, i.e. the median of the ages derived from the SDF tracks. As long as the shape of the age distribution due to the artificial spread in $L_{\mathrm{bol}}$ is at least similar for all star forming regions, we can assume that the median of the measured ages is in some close and unique relation with the real median age. Figure 1 shows the distribution of individual stellar ages, as derived in the following section, for each of the six PMS stellar groups considered here. Also indicated are the median values which will be used in the following.

\section{The star forming regions and open clusters}

We now discuss the eight stellar groups taken in consideration, describing the stellar samples and the analyses performed, along the lines sketched in the previous section, in order to make the results comparable to each other. We proceed in chronological order, starting from the youngest stellar association, according to the sequence suggested by Fig. 1. 


\section{1. $\rho$ Ophiuchi}

$\rho$ Ophiuchi, with an age of $\$ 10^{6}$ years, is the youngest region here considered. It is a medium size association forming out of a dense molecular cloud, and most of its members are deeply embedded in the cloud. It has been therefore studied with more success in the near IR and X-ray bands (e.g. Luhman \& Rieke 1999; Imanishi et al. 2001a). Our source of optical/IR data for $\rho$ Ophiuchi members is the work by Luhman \& Rieke (1999) who obtained NIR spectroscopy and photometry of 114 stars and derived, for 69 of these, effective temperatures, $J$ band extinctions and bolometric luminosities. We restrict our analysis to the field of view (FOV) of the two Chandra X-ray observations described below, so lowering the census of our well characterized members (i.e. with $T_{\text {eff }}$ and $L_{\text {bol }}$ ) to 51 . We estimated masses and ages for 43 of these stars using SDF evolutionary tracks, the remaining eight having apparently masses lower than the lowest mass for which SDF tracks are available $\left(0.1 M_{\odot}\right)$. These 43 stars comprise our reference sample for $\rho$ Ophiuchi.

\subsubsection{X-ray data and analysis}

We have retrieved, from the Chandra Data Archive (http://asc.harvard.edu/cda/), two deep ( $\approx 100 \mathrm{ksec}$ each) and slightly overlapping ACIS-I observation of the $\rho$ Ophiuchi cloud (Obs. Id. 635, 637). A thorough analysis of the first observation has been already reported by Imanishi et al. (2001a), focusing mainly on medium resolution X-ray spectra and on source variability; Imanishi et al. (2001b) have used both observations to study X-ray emission of the known brown dwarfs and candidates brown dwarfs in the region. However, Imanishi et al. (2001b) do not produce a list of sources and Imanishi et al. (2001a) do not compute upper limits to X-ray luminosities of undetected cluster members, a necessary step in order to avoid selection effects. To uniform our analysis to the general principles stated in Sect. 2, we re-analyzed the two dataset.

First, in order to selectively lower the background level we filtered the two event files retaining only events with energies in the 0.3 to $8.0 \mathrm{keV}$ range, likely containing the largest fraction of the X-ray flux from $\rho$ Ophiuchi members. We then detected sources and measured count rates ${ }^{4}$ using PWDETECT (Damiani et al. 1997; Damiani et al., in preparation $)^{5}$ and a signal to noise ratio threshold of 5.10, which, given the background level of the two observations, is expected to yield $\sim 1$ spurious detection in each FOV. Ninety sources were detected in obs. 635 and 77 in obs. 637, after removal from the latter source list of $\sim 20$ spurious detection due to a bright CCD column in coincidence with a luminous X-ray source.

\footnotetext{
${ }^{4}$ Effective exposure times were taken from an exposure map computed using the tools in the Chandra Interactive Analysis of Observations (cIAo) package (http://asc.harvard.edu/ciao/), for an incident energy of $2.16 \mathrm{keV}$. The choice of energy is however not critical as vignetting depends only mildly on energy for the range of energies relevant to stellar coronae.

${ }^{5}$ See also http://www . astropa.unipa.it/progetti_ricerca/ PWDetect
}

For comparison Imanishi et al. (2001a) detect 87 sources in obs. 635. Seven sources located in the small overlap region of the two observations $\left(\sim 40 \operatorname{arcmin}^{2}\right)$, were detected in both observations. In the same region 6 sources appeared instead in only one of the two datasets. We created a merged source list, assigning to the sources detected in both observations uncertainty-weighted averages of count rates and spatial coordinates. Our merged source list comprises 160 distinct X-ray sources with count rates ranging from $6.3 \times 10^{-5}$ to $0.79 \mathrm{cnts} \mathrm{s}^{-1}$.

We cross-identified our detected X-ray sources with the list of 43 selected $\rho$ Ophiuchi members discussed in the previous section. For comparison purposes (see below), we have also identified, with the same list of members, the X-ray sources detected by Imanishi et al. (2001a). In both cases the identification process went smoothly, thanks to the high spatial resolution of Chandra and the relatively low spatial density of objects in the region. We identify 36 members with $35 \mathrm{X}$-ray sources, a close pair of members (GY240A and GY240B) being not resolved in the X-ray image (we treat the measured count-rate of the pair as an upper limit to the count-rate of each star). For the seven members undetected in the ACIS-I data we computed upper limits to count rates using PWDETECT and the same signal to noise ratio threshold used for detections.

We estimate X-ray luminosities of stars in our sample from ACIS-I count rates and assuming a distance of 165 pc (Dame et al. 1987) ${ }^{6}$. Count rates to flux conversion factors were computed, as described in Sect. 2.1, i.e. taking $k T=2.16 \mathrm{keV}$ and $N_{\mathrm{H}}$ proportional to the $J$ band absorption, $A_{J}$ (see below). Thanks to the analysis of medium resolution ACIS-I spectra performed by Imanishi et al. (2001a), which also yielded independent estimates of X-ray luminosities, we are in the position to check these assumptions.

The median(mean) of the quiescent state $k T$ derived from spectral fits (Imanishi et al. 2001a) for 28 stars in our member list is $2.1(2.3) \mathrm{keV}$, indeed similar to our value. Moreover, considering the relevant uncertainties of these best fit $k T$, their distribution is consistent with a narrow range of values: $\sim 50 \%$ fall inside the $k T=1.5-2.5 \mathrm{keV}$ range and another $35 \%$ have 90\% error bars consistent with the same interval.

In regard to extinction, Fig. 2 shows the proportionality between X-ray derived $N_{\mathrm{H}}$ and $A_{J}$. The proportionality constant was assumed to be the median of the measured $N_{\mathrm{H}} / A_{J}: 4.72 \times$ $10^{21}$. This is somewhat lower than what would be expected on the basis of a normal optical/IR extinction curve (Mathis 1990), $A_{V} / A_{J}=3.55$, and the relation between $N_{\mathrm{H}}$ and $A_{V}$ given either by Ryter (1996), $N_{\mathrm{H}}=2.0 \times 10^{21} A_{V}$ or Predehl $\&$ Schmitt (1995), $N_{\mathrm{H}}=1.79 \times 10^{21} A_{V}$. With these assumptions we would have derived $N_{\mathrm{H}} / A_{J}=\left(N_{\mathrm{H}} / A_{V}\right)\left(A_{V} / A_{J}\right)=$ $7.1 \times 10^{21}$ and $6.35 \times 10^{21}$ respectively, i.e. about $30-50 \%$

${ }^{6}$ There is some uncertainty in this distance: an Hipparcos based estimate of the distance to the Upper Scorpius region, of which the $\rho$ Ophiuchi cloud is part, indicates $\sim 145$ pc (de Zeeuw et al. 1999). Had we adopted this value $L_{\mathrm{X}}$ would be lowered by $\sim 0.12$ dex. The $L_{\mathrm{X}} / L_{\mathrm{bol}}$ values, on which our main conclusions are based would remain unaffected. Note also that the median stellar age of the region members would increase by $\sim 0.17 \mathrm{dex}$, becoming almost equal to that of the ONC Trapezium region (Sect. 3.2). 


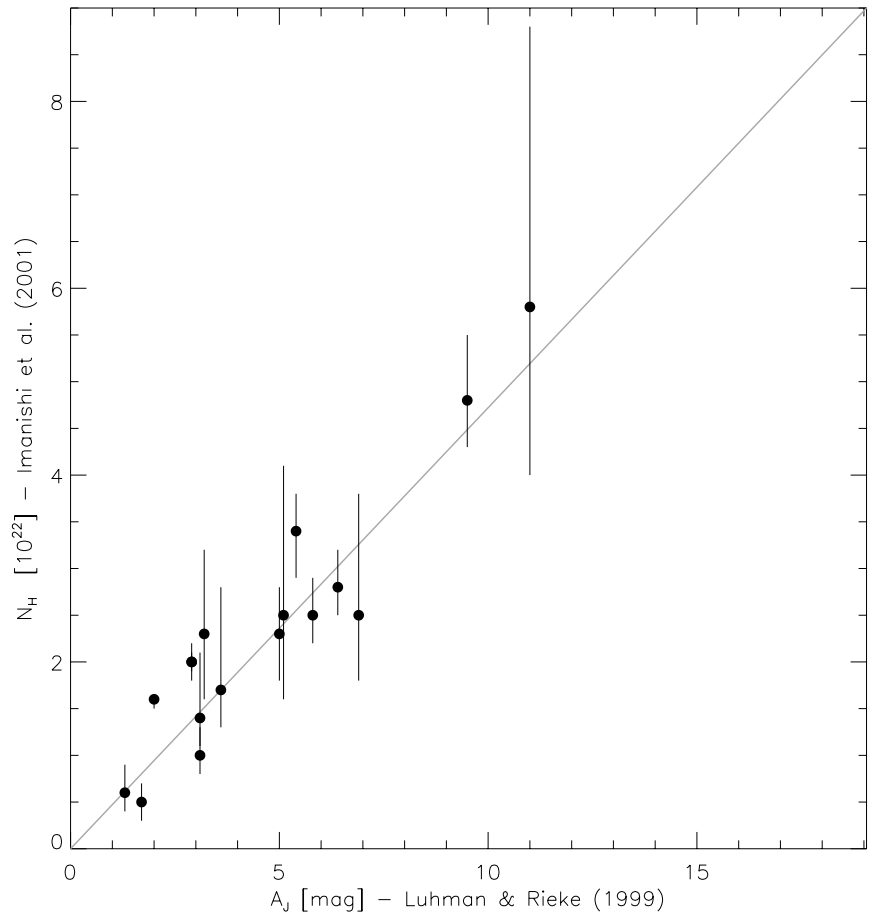

Fig. 2. $N_{\mathrm{H}}$ derived from fittings of X-ray spectra (Imanishi et al. 2001a) vs. $J$ band extinction, $A_{J}$, measured for the same stars by Luhman \& Rieke (1999). The straight line corresponds to the median of the $N_{\mathrm{H}} / A_{J}$ ratios: $4.72 \times 10^{21}$. Error bars are $90 \%$ confidence intervals derived from spectral fits.

higher than our value. This would have in turn resulted in overestimating the conversion factors (and $L_{\mathrm{X}}$ ) by $0.1-0.2 \mathrm{dex}$, depending on absorption in the $A_{V}=2-50$ range. The departure from the normal relation may points toward a peculiarity of the extinction law in the $\rho$ Ophiuchi cloud (cf. Vrba et al. 1993; Kenyon et al. 1998).

Finally, Fig. 3 compares our X-ray luminosities with those estimated by Imanishi et al. (2001a) on the basis of spectral fittings; $90 \%$ confidence interval are also shown for the latter. More than one estimate of $L_{\mathrm{X}}$ is given by Imanishi et al. (2001a) for some variable sources corresponding to quiescent and flaring states (indicated with different symbols in the figure) while we only derive an averaged $L_{X}$ over the whole observations. In general, the estimates for quiescent $L_{X}$ by Imanishi et al. (2001a) and our estimates are fully compatible, with the possible exception of some flaring sources, for which the difference can be easily attributed to the presence of the flare.

We conclude that, at least for $\rho$ Ophiuchi stars, the assumption of a single plasma temperature for all sources and of optical extinctions to calculate conversion factors yields realistic values of $L_{\mathrm{X}}$. From the dispersion of spectral-fit $k T$ and $N_{\mathrm{H}}$ about our assumed values, we estimate that the mean error on our conversion factors is of the order of $0.1 \mathrm{dex}$.

\subsubsection{Activity vs. mass}

We study the relation between activity and stellar mass using the same method described in Flaccomio et al. (2003b) for the Orion Nebula Cluster. Figure 4 shows X-ray luminosity functions for the stars in five mass bins. The same figure shows the

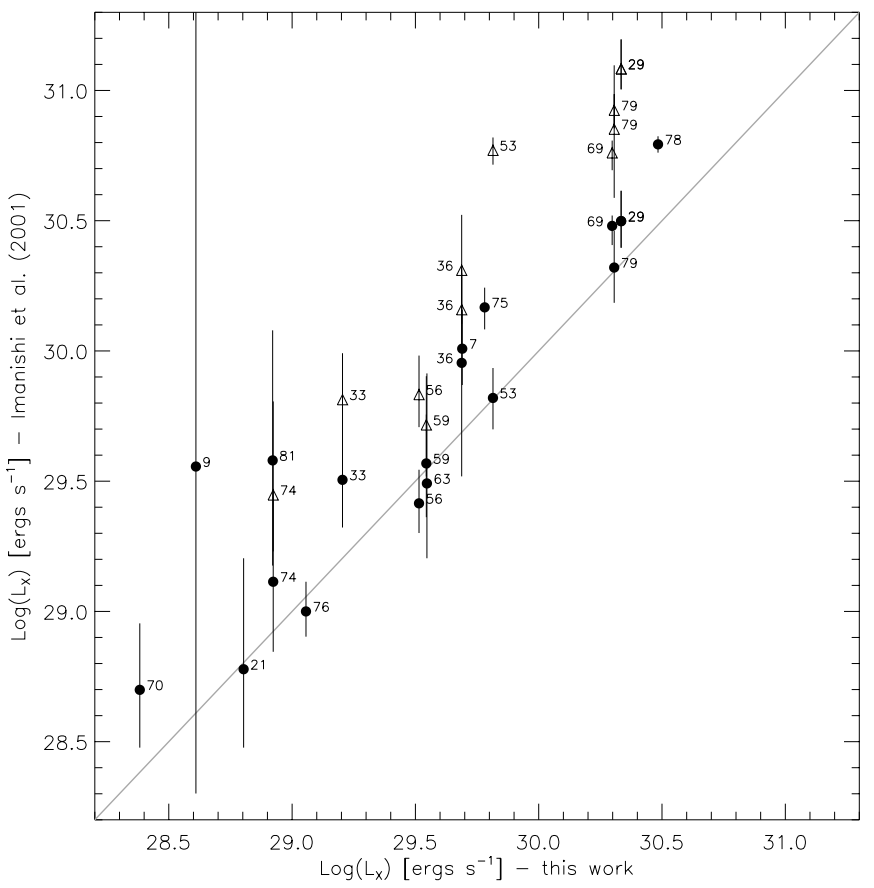

Fig. 3. Comparison of X-ray luminosities estimated from the same ACIS-I data in this work and by Imanishi et al. (2001a). Filled circles indicate that the $L_{X}$ estimate by Imanishi et al. (2001a) refer to the quiescent source emission, while empty triangles refer to flares. The numbers beside each point is the source identification number given by Imanishi et al. (2001a). Note that up to three estimates (one quiescent and two flares) are given for each source. Error bars are $90 \%$ confidence intervals derived from spectral fits.

median $L_{X}$ and the dispersion (25 and $75 \%$ quantiles) of the luminosity functions as a function of mass. Figure 5 shows the same kind of analysis for our second activity indicator: $L_{\mathrm{X}} / L_{\mathrm{bol}}$. We draw the following conclusions: 1$)$ the median $L_{\mathrm{X}}$ is correlated with mass; 2$)$ the median $L_{\mathrm{X}} / L_{\mathrm{bol}}\left(10^{-4.5}-10^{-4.0}\right)$ is significantly below the canonical saturation level, $10^{-3}$; 3) $L_{\mathrm{X}} / L_{\text {bol }}$ is correlated with mass, at least at for $M \lesssim 1 M_{\odot}$. Note that the correlation of $L_{\mathrm{X}} / L_{\mathrm{bol}}$ with mass was also found in the ONC by Flaccomio et al. (2003b): here however the dependence appears to be somewhat steeper.

\subsection{Orion Nebula Cluster}

The X-ray (Chandra HRC) data for the ONC are described in detail in Flaccomio et al. (2003a). There we also defined an optically selected, extinction limited sample of well characterized ONC members (there referred to as the optical sample) that we also use here.

For the present purpose, i.e. the study of the evolution of activity in the PMS, we split the region surveyed by the HRC observation in two sub-areas: the central $5^{\prime} \times 5^{\prime}$, roughly identifiable with the so called Trapezium region, and the remaining of our HRC FOV. It has already been noted (e.g. Hillenbrand 1997) that the Trapezium region is of somewhat more recent formation respect to the outer part of the molecular cloud. Figure 1 shows the cumulative age distribution for the two regions: it is evident that, although the two distributions 
overlap there is an excess of very young stars in the Trapezium. Part of the overlap may be due, other than to the artificial and real age spread in the two regions, to the fact that the area we identify with the Trapezium, likely contains, for a projection effect, a number of stars that are in fact located in front of the Trapezium and should belong more properly to the outer region. In any case, following the discussion in Sect. 2 we will assume that the median activity levels of stars in these two regions are representative of the two median stellar ages.

Figures 4 and 5 show our standard analysis of activity vs. mass for the two spatially segregated regions. We note that the same qualitative trends found for the whole ONC by Flaccomio et al. (2003b) are retrieved in each of the two sub-regions.

\subsection{NGC 2264}

A recent analysis of stellar activity in NGC 2264 was presented by Flaccomio et al. (2003c); these authors utilized published X-ray and optical data (Flaccomio et al. 2000; Rebull et al. $2002 \mathrm{~b}$ ) to investigate the relation between circumstellar environment and activity. The data were there reanalyzed following the general criteria stated in Sect. 2. Here we use the same data and the same reference stellar sample. The sample comprises 178 candidate members from the spectroscopic sample of Rebull et al. (2002b), to which Flaccomio et al. (2003c) were able to assign masses, ages and X-ray luminosities (or upper limits), these latter derived from the ROSAT HRI count rates listed by Flaccomio et al. (2000).

Figures 4 and 5 show our analysis of $L_{\mathrm{X}}$ and $L_{\mathrm{X}} / L_{\text {bol }}$ vs. stellar mass. Given the large number of upper limits, it is quite clear that the sensitivity of the presently available X-ray data (ROSAT HRI from Flaccomio et al. 2000) is not sufficient to define the low luminosity tails of the X-ray luminosity functions so that the median $L_{\mathrm{X}}$ is defined only in two mass bins and the trend of $L_{X}$ with mass seen for the other regions is not so apparent here. The median $L_{\mathrm{X}} / L_{\mathrm{bol}}$ shows a pattern similar to the other regions, being close to the saturation level for $M \lesssim 1.0 M_{\odot}$ and declining for larger masses.

\subsection{Chamaeleon I}

The Chamaeleon I association, with an age of 5-6 Myrs, is the second oldest star forming region considered in this study. Like for NGC 2264, the optical and X-ray data we use here are described in detail by Flaccomio et al. (2003c), where the activity/disk connection was analyzed. The original data that work is based on were taken for the most part from Lawson et al. (1996), but reanalyzed consistently with what done for the other SFRs.

Figure 4 shows the X-ray luminosity functions in our chosen mass ranges and the $L_{\mathrm{X}}$ vs. mass scatter plot. Figure 5 refers, in the same format, to $L_{\mathrm{X}} / L_{\mathrm{bol}}$. The general trend of increasing $L_{X}$ with increasing mass, seen in the other star forming regions, can be clearly observed here. $L_{\mathrm{X}} / L_{\text {bol }}$ seems to be close to the saturation level at all masses. In particular we note that, respect to the younger association, $\rho$ Ophiuchi and the ONC, we do not observe a decrease of the median $L_{\mathrm{X}} / L_{\mathrm{bol}}$ at very low masses.

\section{5. $\eta$ Chamaeleontis}

$\eta$ Chamaeleontis is a small, nearby (97 pc) and relatively old ( $\sim 10 \mathrm{Myr}$ ) PMS cluster discovered through ROSAT X-ray observations by Mamajek et al. (1999). The X-ray properties of 12 members have been investigated by Mamajek et al. (2000), while Lawson et al. (2001) report optical photometry and derive rotational periods. The total $\eta$ Chamaeleontis known population counts 15 stars: 3 are early type stars (one of which, HD 75505 is undetected in the X-rays), 11 are WTTS and 1 is a CTTS. Ten of the 12 low mass members have been discovered through detection in the X-rays (Mamajek et al. 1999). The following optical search for additional members conducted by Lawson et al. (2002) resulted in the discovery of only two non X-ray selected members, among which the CTTS (ECHA J0843.3-7905).

In the following, reassured by the high detection efficiency among the known low-mass cluster population, we will only consider X-ray detected members. The X-ray (ROSAT HRI) count rates are taken from Mamajek et al. (2000) while the optical data, $T_{\text {eff }}$ and $L_{\text {bol }}$, come from Lawson et al. (2001). Following the previous investigations we assumed zero absorption along the line of sight. The determination of masses, ages and X-ray luminosities followed the principles stated in Sect. 2.

Figure 4 shows the X-ray luminosity functions in our mass ranges and the trend of median $L_{X}$ with mass. Figure 5 refers, in the same format, to $L_{\mathrm{X}} / L_{\mathrm{bol}}$. We note that, notwithstanding the low number of stars, the data points are compatible with the same qualitative results found for low mass stars in the slightly younger Chamaeleon I region: a dependence of $L_{\mathrm{X}}$ on mass and a constant "saturated" $L_{\mathrm{X}} / L_{\mathrm{bol}}$.

\subsection{The Pleiades}

The Pleiades open cluster, with an age of $~ 100$ Myr (Meynet et al. 1993) and a distance of only $\approx 127 \mathrm{pc}$, is possibly the best studied young ZAMS cluster in the solar neighborhood. Its stellar population has been nearly completely identified and several X-ray studies performed with ROSAT (e.g. Stauffer et al. 1994; Micela et al. 1996; Micela et al. 1999) have measured X-ray luminosities for a representative fraction of Pleiades members. Here we use this wealth of information for the purpose of extending our study of the evolution of activity from the premain-sequence to the ZAMS.

Our sample of Pleiades members includes 500 stars listed in the open cluster database of C. F. Prosser and J. R. Stauffer ${ }^{7}$ and that fall in the FOV of the X-ray observations described below. We estimate stellar masses from the theoretical SDF isochrone and $V_{0}$ magnitudes as explained in Sect. 2.2. The choice of using the $V$ magnitude instead of, for example, the $B-V$ color as a mass indicator is due to the fact that $V$ is available for all the stars in our sample (vs. $\sim 54 \%$ for $B-V$ ).

\footnotetext{
${ }^{7}$ http://cfa-www.harvard.edu/ stauffer/opencl/ index.html
} 

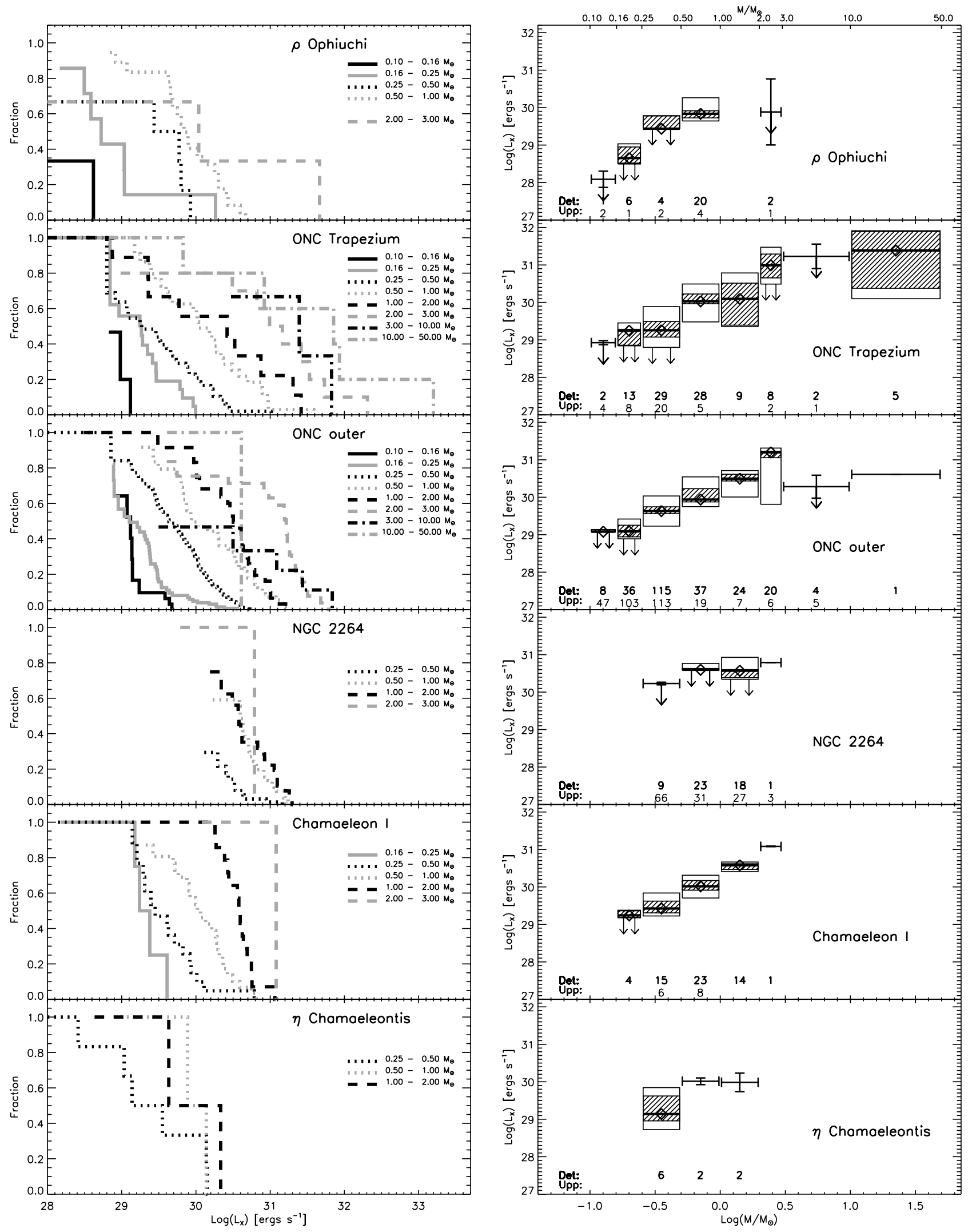

Fig. 4. Left: X-ray luminosity functions (XLF) for our PMS associations in several mass ranges. Right: Median and scatter of log $\left(L_{X}\right)$ as a function of $\log \left(M / M_{\odot}\right)$. Medians are indicated by diamonds centered on thick segments, with uncertainties indicated by the shaded boxes, and the $25 \%$ and $75 \%$ quantiles of the XLF by thin-lined boxes. Two small downward-pointing arrows at box bottom indicate an upper limit on the lower error in median and/or the lower quartile. In some cases medians are substituted by averages (either measured or upper limits), indicated by error bars. Numeric values above abscissa for each box indicate numbers of detections ("Det.") and upper limits ("Upp.") contributing to the XLFs shown on the left. 

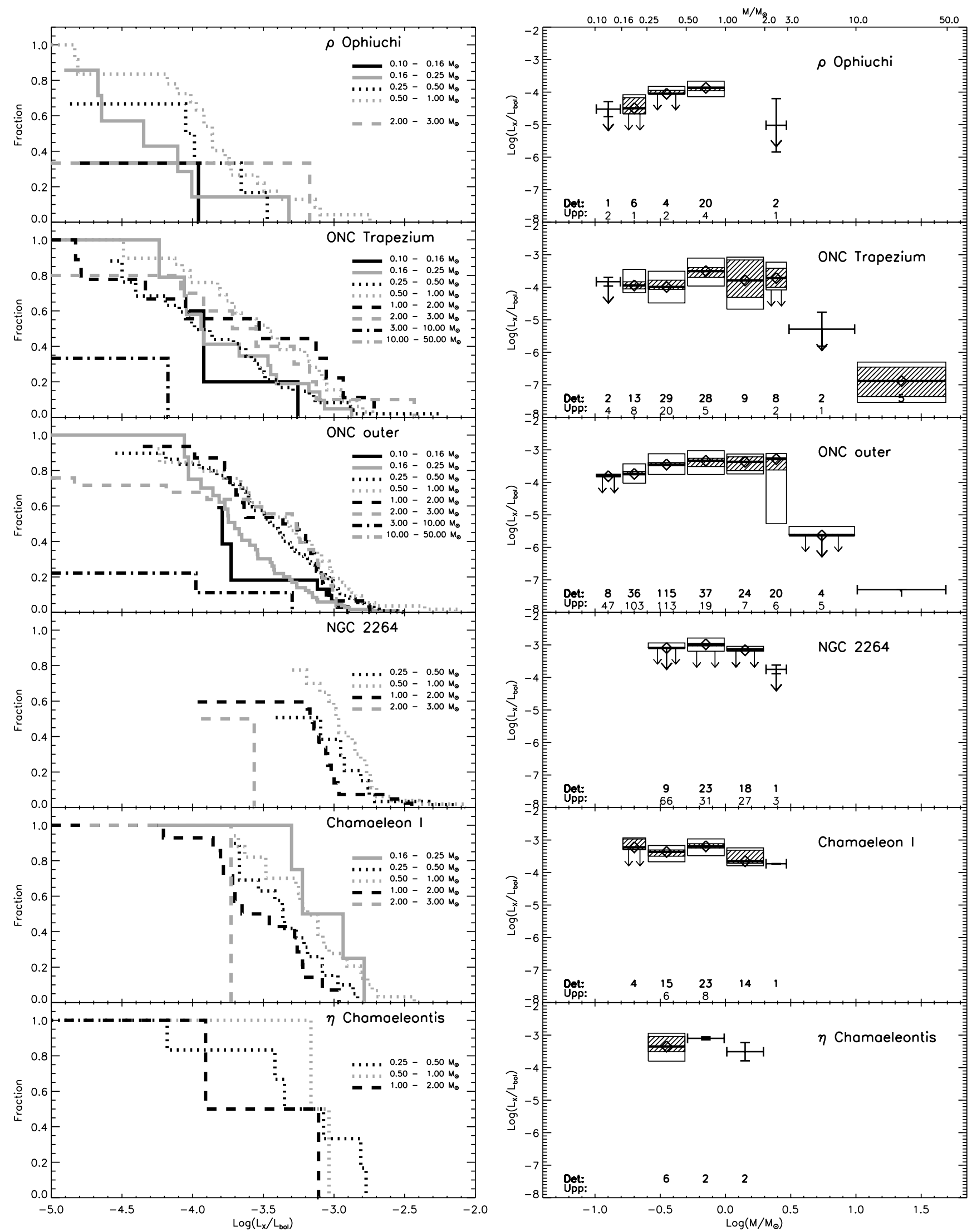

Fig. 5. Same as Fig. 4 but for $L_{\mathrm{X}} / L_{\text {bol }}$ instead of $L_{\mathrm{X}}$. In the scatter plots, diamonds with downward-pointing arrows indicate upper limits to the medians. 
We exclude from the following analysis 38 stars that, in the $V_{0}$ vs. $(B-V)_{0}$ diagram, are more distant than 1 magnitude from the isochrone (along the $V_{0}$ axis). We note that, given the non-perfect adherence of the observational member locus to the SDF isochrone and to the presence of unresolved multiple systems (having lower $V_{0}$ than those pertaining to the primary alone) our mass estimates are somewhat uncertain. We get a rough idea of the uncertainties on mass estimates by comparing our masses derived from $V_{0}$ with those obtained from the same isochrone through $(B-V)_{0}$. The difference in the individual masses are typically smaller than $0.05 \mathrm{dex}$, small compared to the width of the mass ranges used to compute the Maximum Likelihood distributions of $L_{\mathrm{X}}$ and $L_{\mathrm{X}} / L_{\mathrm{bol}}$.

Bolometric luminosities, used for computing $L_{\mathrm{X}} / L_{\mathrm{bol}}$, are estimated, in the same way as masses, from the theoretical isochrone.

$\mathrm{X}$-ray data are taken from the published analysis of 16 different X-ray exposures performed with ROSAT, 8 using the PSPC Micela et al. (1998) and 8 using the HRI (Micela et al. 1999). Each star in our reference catalog was observed between one and nine times ( $\sim 3$ times on average). However, possibly due to sensitivity limits, only $46 \%$ of the stars in our reference list are detected, while for the remaining ones only upper limits to the X-ray luminosities are available.

The estimates of X-ray luminosities we use are taken from Micela et al. (1998) for the PSPC data and Micela et al. (1999) for the HRI data. Both refer to the $0.1-2.5 \mathrm{keV}$ spectral band and are based on the following assumptions: a coronal temperature $k T=0.8 \mathrm{keV}$ and absorption $N_{\mathrm{H}}=2 \times 10^{21} \times$ $3.1 \times E(B-V)$, where $E(B-V)$ is measured individually for about $15 \%$ of the stars, while for the remaining ones a mean value, 0.04 , is used. The spectral band luminosities refer to is narrower than our standard $0.1-4.0 \mathrm{keV}$ band; anyway, because of the low coronal temperature of Pleiades sources, the difference in conversion factor due to the difference in band is small: for $k T=0.8 \mathrm{keV}$, the fraction of emitted energy falling in the 2.5-4.0 keV band, and therefore the amount by which we underestimate $L_{\mathrm{X}}$, is $\lesssim 0.01 \mathrm{dex}$. Only for $k T \sim 1.5 \mathrm{keV}$, hotter than typical MS coronal sources (Gagné et al. 1995b; Daniel et al. 2002), the difference in $L_{X}$ due to the narrower spectral band reaches $\sim 0.05$ dex. Softer spectra, $k T \sim 0.4-0.5 \mathrm{keV}$, have been inferred for main sequence $F$ type stars (Gagné et al. 1995b; Flaccomio 2002; Daniel et al. 2002): assuming $k T=0.8 \mathrm{keV}$ results in this case in an overestimation of $L_{\mathrm{X}}$ of up to $\sim 0.05$ dex, which is small compared to the other sources of random uncertainties (e.g. counting statistic and distance spread). The assumption of an average value of absorption, for stars for which no individual measurement of $E(B-V)$ is available, is also justified: $80 \%$ of the stars for which $E(B-V)$ has been measured have a corresponding $N_{\mathrm{H}} \lesssim 10^{21} \mathrm{~cm}^{-2}$, which, for $k T=0.8 \mathrm{keV}$ translates into a difference on the individual conversion factor of $\lessgtr 0.1$ dex respect to assuming the mean absorption. Moreover, while stars that have individual extinction estimates are located in a sky area of high nebulosity (this being the reason that prompted the measurement of individual extinctions), the remaining stars are located in an area apparently devoid of clouds and most likely have fairly uniform $E(B-V)$; the former estimate of 0.1 dex for the uncertainty on the conversion factor is therefore most likely too pessimistic.

Given the multiplicity of observations, in order to assign a single $L_{\mathrm{X}}$ value to each of our stars, we proceeded as in Flaccomio et al. (2000), i.e. we compute maximum likelihood mean luminosities using all the available information (detections and upper limits). This was implemented by computing, with the ASURV package (Feigelson \& Nelson 1985), the Kaplan Mayer estimator of the values of $\log \left(L_{\mathrm{X}}\right)$ available for each source. Because of the time variable nature of X-ray emission, the thus derived mean $\log \left(L_{\mathrm{X}}\right)$ of $\sim 6 \%$ of the stars which were detected in some of the observations turned out to be upper limits. Out of the 483 stars in our reference sample we therefore have estimates of mean $\log \left(L_{\mathrm{X}}\right)$ for $40 \%$ of the stars and upper limits for the remaining ones.

Figure 6 shows X-ray luminosity functions in our chosen mass bins and the $\log \left(L_{\mathrm{X}}\right)$ vs. mass scatter plot. The same analysis is repeated for $\log \left(L_{\mathrm{X}} / L_{\mathrm{bol}}\right)$ in Fig. 7. Low mass stars $\left(\log \left(M / M_{\odot}\right) \lesssim-0.3\right)$ appear to be saturated, with $\log \left(L_{\mathrm{X}} / L_{\mathrm{bol}}\right) \sim-3$, and show the consequent mean correlation of $L_{X}$ with mass. Higher mass stars however are not saturated, so that the relation of luminosity with mass is lost, as also evinced by the onset of the activity - rotation connection which is indeed observed at this mass boundary (cf. Micela et al. 1999).

\subsection{NGC 2516}

The open cluster NGC 2516 is in many respects similar to the Pleiades, and it has indeed been often named the southern Pleiades. It is however slightly older, 140 Myrs (Meynet et al. 1993), and more distant (387 pc, Jeffries et al. 1997).

Our data are based on the photometric survey of Jeffries et al. (2001) and the analysis of eight Chandra X-ray observations reported by Damiani et al. (2003). Our initial member list is the same as that used by Damiani et al. (2003) and comprises 725 stars indicated as members by Jeffries et al. (2001) and falling in the FOV of at least one of the Chandra observations. Stellar masses, and bolometric luminosities, were derived analogously with what described for the Pleiades cluster from $V_{0}$ magnitudes and a theoretical (SDF) isochrone, the only difference being in the choice of the isochrone appropriate to the larger age of NGC 2516. We excluded from our reference sample 52 stars that, in the $V_{0}$ vs. $(B-V)_{0}$ diagram, lie farther away than 1 mag (along the $V_{0}$ axis) from the $140 \mathrm{Myr}$ isochrone and four stars that were found to be outside the $M_{V}$ range covered by the isochrone.

We adopt X-ray luminosities from Damiani et al. (2003). These are averages of the luminosities estimated from detected source counts in all the analyzed observations. Conversion factors between counts and luminosity in the $0.1-4.0 \mathrm{keV}$ band are there computed assuming uniform extinction, $N_{\mathrm{H}}=$ $8.2 \times 10^{20}$, and, for bright sources, a two temperature spectral model (one of which fixed at $0.26 \mathrm{keV}$ ), constrained by observed hardness ratios. For weaker stellar sources and upper limits, the conversion factor is taken as the median of the values derived for bright sources with the same spectral type. 
Table 1. Optical and X-ray data for the stellar sample.

\begin{tabular}{cccccc}
\hline \hline N. & Region & $\begin{array}{c}\text { Mass } \\
{\left[M_{\odot}\right]}\end{array}$ & $\begin{array}{c}\log (\text { Age }) \\
\text { [yr. }]\end{array}$ & $\begin{array}{c}\log \left(L_{\mathrm{X}}\right) \\
{\left[\operatorname{ergs~s}^{-1}\right]}\end{array}$ & $\log \left(L_{\mathrm{X}} / L_{\text {bol }}\right)$ \\
\hline$(1)$ & $(2)$ & $(3)$ & $(4)$ & $(5)$ & $(6)$
\end{tabular}

Notes - Column 1: sequential reference number; Col. 2: identifier of the region the star belongs to.

The Table, comprising 2129 rows, is available in electronic form at http://www. edpsciences.org

Damiani et al. (2003) estimate that, given the range of variation of the conversion factors derived from hardness-ratios, the uncertainty in individual $\log \left(L_{\mathrm{X}}\right)$, introduced by an inaccurate spectral model, is at most $\sim 0.15$ dex.

Figures 6 and 7 show our standard analysis of activity, $L_{\mathrm{X}}$ and $L_{\mathrm{X}} / L_{\mathrm{bol}}$, as a function of mass. The same qualitative trends observed for the Pleiades are retrieved here.

\section{Evolution of coronal activity}

We now utilize the data presented in the previous section to study the evolution of stellar activity in specific stellar mass ranges. Table 1, available in electronic form, summarizes the data described in Sect. 3 and also utilized in the following. For our purpose (see Sect. 2.2) we assume that the median activity level of each roughly coeval PMS stellar group is representative of the PMS activity at the median of stellar ages in the same group.

Figure 8 shows, in the left hand column, for each of our chosen mass ranges, the median $\log \left(L_{X}\right)$ of each of the eight regions studied in the previous section as a function of median association age. The same analysis is presented for $\log \left(L_{\mathrm{X}} / L_{\mathrm{bol}}\right)$ in the right hand column. In order to assess the significance of the difference between $L_{\mathrm{X}}$ and $L_{\mathrm{X}} / L_{\mathrm{bol}}$ distributions of stars in a given mass range and belonging to different regions (i.e. having different ages) we have performed two population statistical tests using the ASURV package. ASURV performs five different tests that give quantitatively different results. In comparing distribution of activity indicators we will consider, quite arbitrarily, significantly and possibly different two samples for which the majority of the tests give lower than, respectively, $1 \%$ and $10 \%$ probability that the samples are not drawn from the same parent population. In order to further assess the presence of correlations between median activity and age of the PMS regions we have also performed two correlation tests (namely the Cox proportional hazard model test and the generalized Kendall's $\tau$ test), also using ASURV.

Summarizing the trends for $\log \left(L_{\mathrm{X}}\right)$ on the left side of Fig. 8 as well as the results of the tests, we have weak evidence of an increase of $\log \left(L_{\mathrm{X}}\right)$ in the PMS, for $M<0.25 M_{\odot}$. More specifically, in the $M=0.16-0.25 M_{\odot}$ mass bin, $\rho$ Ophiuchi $(\log ($ Age $)=5.99)$ may have lower levels respect to the outer ONC $(\log ($ Age $)=6.28)$ and the Chamaeleon I region $(\log ($ Age $)=6.60)$. In the following two mass ranges there is no evidence for a temporal evolution of median $L_{X}$, with the notable exception of NGC $2264(\log ($ Age $)=6.34)$, for which the medians, or the high luminosity tail of the luminosity functions if the median are not defined, are higher than that of the other regions of similar age (significantly so in the 0.5 $1.0 M_{\odot}$ range). Similar lack of evidence for a PMS evolution is retrieved for the two highest mass bins, although the two population tests indicate a significant lowering of $L_{\mathrm{X}}$ between the age of Chamaeleon I and $\eta$ Chamaeleontis (the two oldest PMS regions) in the $1-2 M_{\odot}$ range. In all mass bins the results of the correlation tests do not evidence any overall trends of $L_{\mathrm{X}}$ with age in the PMS. A significant decrease of $L_{\mathrm{X}}$ from the PMS to the age of the two ZAMS clusters is observed at all masses.

The trends of $\log \left(L_{\mathrm{X}} / L_{\mathrm{bol}}\right)$ are somewhat more definite. Apart from the lowest mass bin, for which the evidence from the two population tests is only at the $90 \%$ level, we observe, for $M<1 M_{\odot}$, a significant trend of increasing $\log \left(L_{\mathrm{X}} / L_{\mathrm{bol}}\right)$ in the early PMS phase followed by a subsequent leveling at the saturation level, $\sim-3$. This is clearly indicated both by the two population tests and by the correlation tests (with the exception of the $0.5-1.0 M_{\odot}$ for which the result of the correlation tests are only at the $\sim 85 \%$ level, increasing to $97 \%$ if we only consider the four youngest regions). The saturation level is likely retained down to $\sim 100 \mathrm{My}$ for $M<0.5-1.0 M_{\odot}$, while at higher masses we observe a decline respect to our oldest PMS region. In the two most massive bins all the tests do not evidence a statistically significant evolution of PMS activity as measured by $L_{\mathrm{X}} / L_{\mathrm{bol}}$.

\section{Discussion}

We now attempt a physical interpretation of our observational results. More specifically we discuss whether the observed activity levels can be described with the same ideas that are successful for more evolved MS stars, i.e. with the mechanism of a stellar dynamo driven by stellar rotation plus convection.

A recent observational study of the dependence of activity on rotation and convection in the MS, has been performed by Pizzolato et al. (2003), who propose two possible descriptions of stellar activity levels.

The first scenario relates the observed X-ray luminosity $\left(L_{\mathrm{X}}\right.$, in ergs/s) with the stellar rotational period ( $P_{\text {rot }}$, in days):

$L_{\mathrm{X}} \approx 10^{30} P_{\mathrm{rot}}^{-2}$

This relation is valid, within uncertainties, for rotational periods such that $L_{\mathrm{X}} / L_{\mathrm{bol}} \lesssim 10^{-3}$ (where $L_{\mathrm{bol}}$ depends, on the MS, on stellar mass), while for shorter $P_{\text {rot }}$ the relation saturates at $L_{\mathrm{X}} \approx 10^{-3} L_{\mathrm{bol}}$. Stars of all masses therefore follow the same relation of $L_{\mathrm{X}}$ with $P_{\text {rot }}$ when activity is not saturated but saturate at different values of $L_{X}$ according to their bolometric luminosities. In this description convection does not enter explicitly, although the presence of convection is necessary for the presence of coronal activity, i.e. the relation holds only for stars that possess a convective envelope.

The second description involves the Rossby number, i.e. the dimensionless ratio between the stellar rotational period and the convective turnover time: $R_{0}=P_{\text {rot }} / \tau_{\text {conv }}$. 

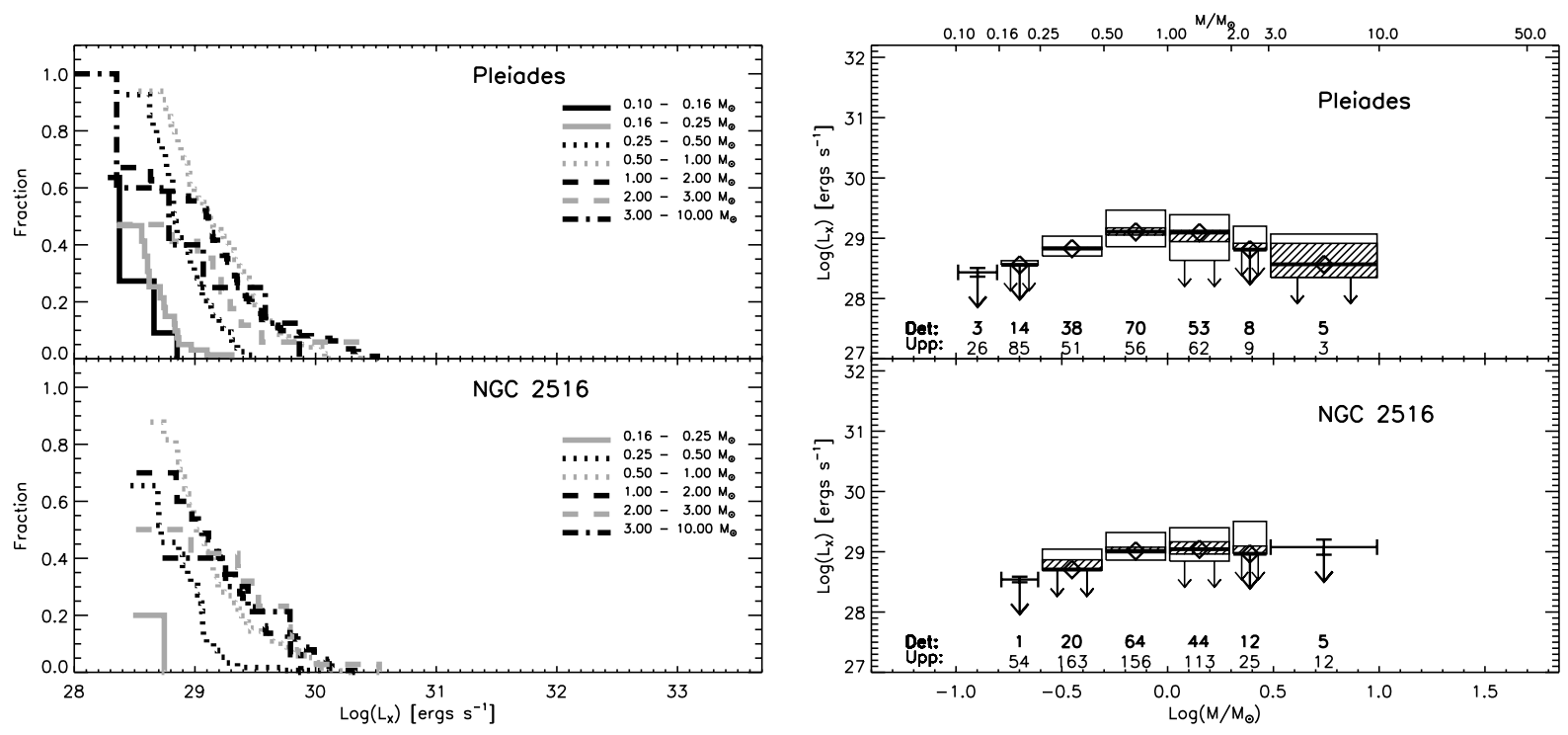

Fig. 6. Same as Fig. 4 for the two ZAMS clusters.
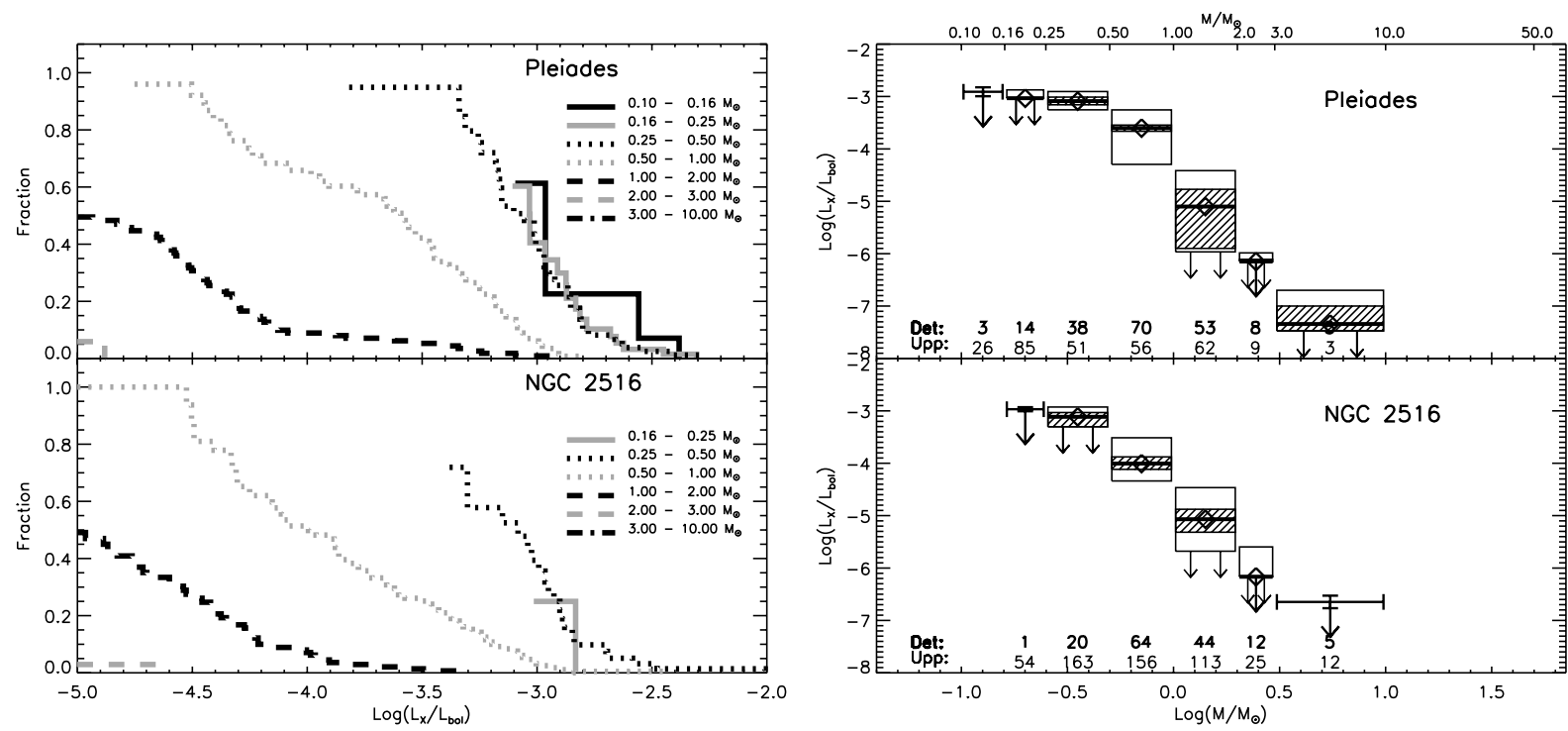

Fig. 7. Same as Fig. 5 for the two ZAMS clusters.

The observational data, for stars in a fairly wide range of masses, can be adequately described by the relation:

$\frac{L_{\mathrm{X}}}{L_{\mathrm{bol}}} \propto R_{0}^{-2}$.

This holds for large enough values of $R_{0}$ and saturates, at $L_{\mathrm{X}} / L_{\mathrm{bol}} \sim 10^{-3}$ for smaller Rossby numbers, i.e. for short rotational periods or long convective turnover times. In this description, like in the previous, stars of all mass follow the same relation in the non-saturated regime; however, contrary to the previous case, here both the saturation level and the value of the independent variable, $R_{0}$, at which saturation occurs are independent from mass. We therefore reach an unifying picture at the expense of the introduction of two additional variables, $L_{\text {bol }}$ and $\tau_{\text {conv }}$.

As shown by Pizzolato et al. (2003) the two descriptions are, in the MS phase, equivalent (within observational uncertainties): the theoretical stellar structure models from which the values of $\tau_{\text {conv }}$ can be derived indicate that, for MS stars in a wide range of mass, the relation $\tau_{\text {conv }} \propto L_{\text {bol }}^{-1 / 2}$ holds, at least approximately, with a proportionality constant that is quite independent of stellar mass. Equation (2) is then approximately equivalent to Eq. (1). By considering only MS stars, it is therefore difficult to establish which of the two relations gives a more accurate description of the data and thus, to shed light on the physical mechanisms responsible for activity and for the saturation phenomena.

In the following we compare these two descriptions to our PMS data. We will try to decide whether any of the two can explain the observations, either entirely or by introducing some ad hoc modification.

Following Pizzolato et al. (2003), we will refer to the PMS evolutionary models of Ventura et al. (1998); these models compute the convective turnover time, whose evolution in the PMS is essential for our study. Treatment of convection is 

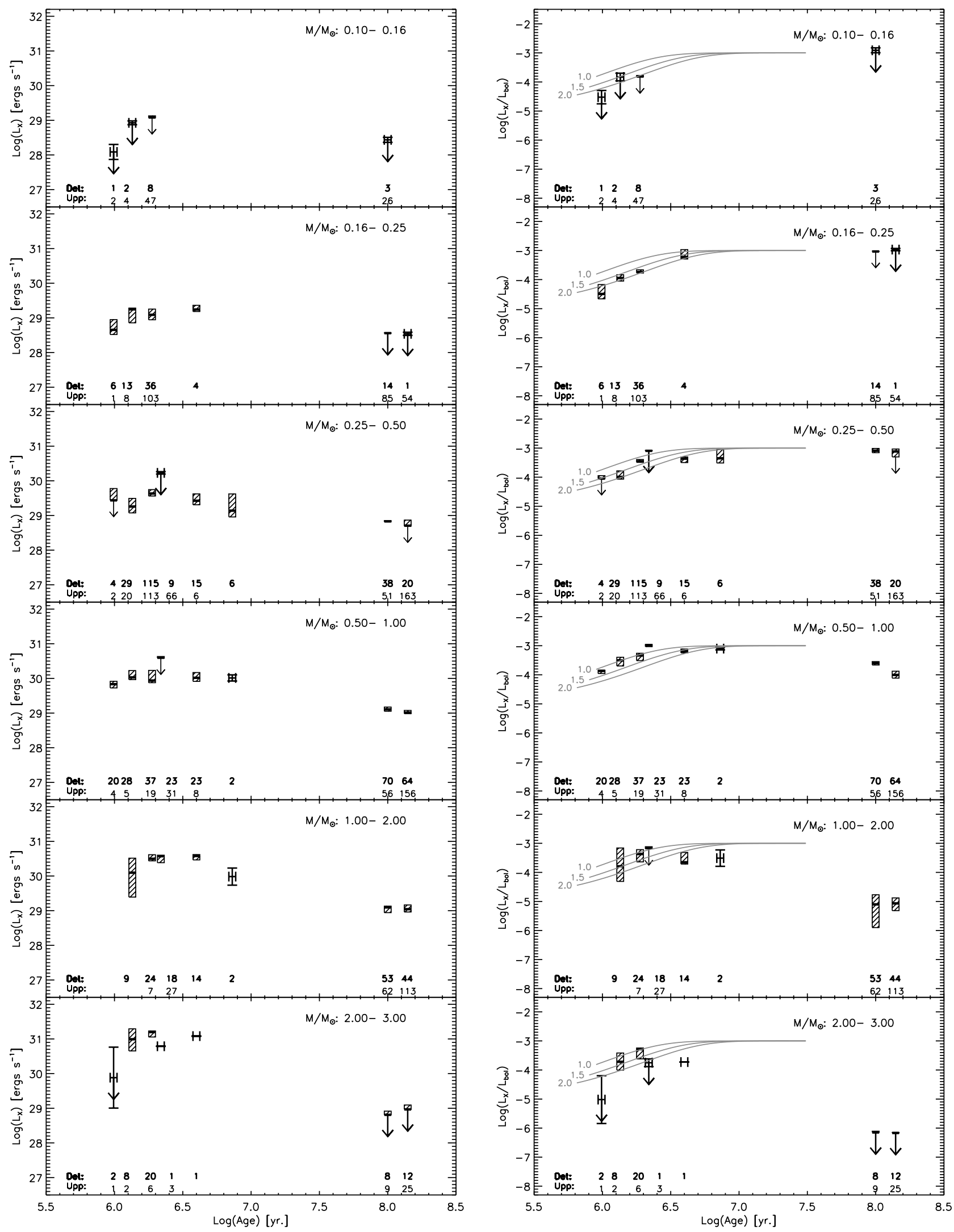

Fig. 8. Time evolution of median $L_{\mathrm{X}}$ and $L_{\mathrm{X}} / L_{\text {bol }}$ (left and right respectively). Medians are represented by small horizontal segments, with hatched boxes representing uncertainties. Down-pointing arrows indicate upper limits on the median and on the lower error (thick and solid arrows respectively). In some cases medians are substituted with averages (either defined or upper limits), indicated by error bars. Numeric values above abscissa indicate numbers of detections ("Det.") and upper limits ("Upp.") in each of the bin. The three curve in the right hand panels refer to three realizations of the simple model described in Sect. 5 , characterized by the disk dissipation time, $\tau_{\text {disk }}$, whose value is reported beside the curves in units of $10^{6}$ years. 
notoriously one of the most difficult points of stellar structure models: Ventura et al. (1998) use two alternative convective theories/approximations: Mixing Length Theory (MLT) and Full Spectrum of Turbulence (FST). For each set of models they compute two differently defined values of $\tau_{\text {conv }}$ : one computed at the bottom of the convective zone (not defined for fully convective stars), the other throughout the whole convective zone. The absolute values of $\tau_{\text {conv }}$ as a function of stellar mass and age depend on its definition and on the choice of convective theory (MLT or FST). However, the trends with mass and age are similar in all cases. In the following, for our argumentation, we will be mainly interested in the qualitative trends of $\tau_{\text {conv }}$ relative to the MS values, so that our conclusions do not depend on the particular model and/or definition. First of all we note that the inverse relation between $L_{\text {bol }}$ (directly related to mass at a given age) and $\tau_{\text {conv }}$ which holds in the MS does not extend unmodified to the PMS. Indeed, according to the Ventura et al. (1998) models, moving to younger ages, a star of a given mass increases both its $L_{\text {bol }}$ and its $\tau_{\text {conv }}$, and this latter is more than one order of magnitude larger, for $1 M_{\odot}$ stars, at the age of the PMS regions considered in this paper, respect to the MS value. The two equations, (1) and (2), that in the MS are equivalent, will then produce in this case different and identifiable predictions.

\subsection{Implications for PMS stars}

We now discuss the implications of the two descriptions of activity summarized above for our PMS clusters, with particular reference to the ONC case, for which a rich sample of rotational periods is available (cf. Herbst et al. 2002). We recall that no relation between activity and rotation has been observed to date in the ONC (Feigelson et al. 2002; Flaccomio et al. 2003b) as well as in the other regions discussed in this work.

The most direct consequence of our first scenario (cf. Eq. (1)) when applied to PMS stars is that, due to the larger bolometric luminosity of these latter respect to MS stars, the saturation level is predicted to be reached at higher values of $L_{\mathrm{X}}$ and smaller rotational periods. Stars with measured rotational periods in the ONC sample ( $P_{\text {rot }}$ up to $\sim 20$ days) should therefore not be saturated. This however contrast with the absence of a $L_{\mathrm{X}}-P_{\text {rot }}$ correlation and with the high, close to saturation, values of $L_{\mathrm{X}} / L_{\mathrm{bol}}$ observed in all our star forming regions and, in particular, for stars more massive than $0.5 M_{\odot}$. For example, excluding the young $\rho$ Ophiuchi members, more than half of the stars in the $0.5-1.0 M_{\odot}$ mass range of each of our star forming regions have $\log \left(L_{\mathrm{X}} / L_{\mathrm{bol}}\right)>-3.5$. Using Eq. (1) and the $L_{\text {bol }}$ values predicted by the SDF evolutionary tracks, we calculate that $\log \left(L_{\mathrm{X}} / L_{\mathrm{bol}}\right)>-3.5$ would imply, for a $M=1.0 M_{\odot}$ star at the age of the ONC, rotational periods shorter than 0.8 days. Likewise, saturated stars $\left(\log \left(L_{\mathrm{X}} / L_{\mathrm{bol}}\right)=-3.0\right)$ should have $P_{\text {rot }}<0.4$ days. However, $\sim 97 \%$ and $\sim 99 \%$ of ONC stars have measured periods longer than 0.8 and 0.4 days respectively. We can then exclude that in the PMS $L_{X}$ is related to $P_{\text {rot }}$ by Eq. (1).

We now turn to Eq. (2) and to the possible role of the convective turnover time, $\tau_{\text {conv }}$. As already noted the
Ventura et al. (1998) models predict that at the age of the ONC and younger, the $\tau_{\text {conv }}$ of solar mass stars is more than 1 order of magnitude larger respect to the MS value. Assuming that Eq. (2) holds and that saturation occurs at the same $R_{0}$ as for MS stars, and recalling that MS solar mass stars saturate at $\log \left(P_{\text {rot }}\right) \sim 0.2$ (with $P_{\text {rot }}$ measured in days, cf. Pizzolato et al. 2003 ), an increase of an order of magnitude in $\tau_{\text {conv }}$ translate in an increase of the same amount in the rotational periods at which saturation occurs. We therefore expect PMS solar mass stars with $\log \left(P_{\text {rot }}\right) \lesssim 1.2$ to be saturated. Given the similarity of convective turnover times of stars of different masses at young ages, saturation periods of the same order are expected for lower mass stars. Now, $\log \left(P_{\text {rot }}\right)=1.2$ corresponds to periods of about 16 days, i.e. longer than typically measured in the ONC. This picture would therefore predict that virtually all PMS stars are saturated. Therefore they should not exhibit a relation of activity vs. rotation and they should all have $\log \left(L_{\mathrm{X}} / L_{\mathrm{bol}}\right) \sim-3$. Such a prediction is almost consistent with the observations: we do not indeed observe a correlation of activity with rotation and for most star forming regions we do observe almost saturated activity levels. This is especially true for the older PMS regions (NGC 2264, the Chamaeleon I and $\eta$ Chamaeleontis associations) and for the more massive stars (cf. Figs. 5 and 8). However, quite clearly, the youngest stars, especially at low masses, show somewhat lower $L_{\mathrm{X}} / L_{\mathrm{bol}}$ levels. For the ONC sample of rotational periods we have no evidence that rotational periods of low mass stars are longer than for more massive stars. On the contrary there is likely an opposite dependence of median $P_{\text {rot }}$ with mass, the median $\log \left(P_{\text {rot }}\right)$ being $\sim 0.4$ (from 15 stars) and $\sim 0.9$ (from 49 stars) in the $0.1-0.16 M_{\odot}$ and $0.5-1.0 M_{\odot}$ mass range, respectively. We therefore conclude that, although Eq. (2), i.e. the relation between $L_{\mathrm{X}} / L_{\mathrm{bol}}$ and $R_{0}$, does better than the relation between $L_{\mathrm{X}}$ and $P_{\text {rot }}$ (Eq. (1)), it does not by itself explain the PMS observations.

One tentative explanation for the low $L_{\mathrm{X}} / L_{\mathrm{bol}}$ stars may invoke the still controversial existence of the super-saturation regime (Randich 2000; Feigelson et al. 2003). There is some evidence of a third regime in the $L_{\mathrm{X}} / L_{\mathrm{bol}}$ vs. Rossby number relation obtained using MS stars, other than the normal inverse relation at large $R_{0}$ and the saturation regime: activity of stars with very low Rossby numbers, i.e. very fast rotations or long convective turn-over times may show a direct correlation with $R_{0}$. The faster average rotation of lower mass stars in the ONC, which indeed have lower average $L_{\mathrm{X}} / L_{\mathrm{bol}}$, seems consistent with such an explanation. However, low mass stars in the ONC span the whole range of measured rotational periods and activity is not correlated, either directly or inversely, with rotational period (cf. Fig. 2 in Flaccomio et al. 2003b); this holds even when the analysis is restricted to narrow mass ranges in order to reduce any possible difference in stellar structure (i.e. $\tau_{\text {conv }}$ ). Moreover the increase in activity in the first few Myr observed at a given mass (Sect. 4) would imply, in this hypothesis, a spin-down of stars in the early PMS. Although theoretical speculations suggest that this could indeed happen due to non-instantaneous disk locking (Hartmann 2002), observational studies of the rotational evolution in the ONC and NGC 2264 (Rebull et al. 2002a) rather suggest that 
stars keep constant period as they evolve down the convective tracks. Given the lack of firm observational evidence of the super-saturation regime, here we do not further explore this hypothesis but rather present and discuss an alternative explanation.

\subsection{The disk hypothesis}

We now introduce a simple model that tries to account for the observed evolution of activity of low mass stars in the early PMS phase. Given the persistent lack of rotational data for the greatest part of our stellar sample, the uncertainties in the X-ray data and in the theoretical evolutionary models, we keep our model extremely simple and, at this point, consider it only as a hint for further observational and theoretical work.

The observational and theoretical bases of our model are the followings:

- Most PMS stars, especially in the older regions and/or not of very low mass have almost saturated activity levels: $\log \left(L_{\mathrm{X}} / L_{\mathrm{bol}}\right) \sim-3.0$. A relation between activity and rotation is generally not observed. As discussed at the end of the last section, the relation of $L_{\mathrm{X}} / L_{\mathrm{bol}}$ with Rossby number (Eq. (2)) suggests that activity should indeed be saturated.

- Stars with circumstellar accretion disks, in several star forming regions, show lower median activity levels (both in $L_{\mathrm{X}}$ and $\left.L_{\mathrm{X}} / L_{\mathrm{bol}}\right)$ respect to those with weaker or no evidence of a disk and/or accretion (Stelzer \& Neuhäuser 2001; Flaccomio et al. 2003b,c). The origin of the lower activity level is at present unknown.

We will make the following assumptions:

1) Departure from saturation is due exclusively to the presence of disks, stars that have already shed their disks having saturated $\log \left(L_{\mathrm{X}} / L_{\mathrm{bol}}\right)$.

$H_{\text {sat. }} \equiv \log \left(L_{\mathrm{X}} / L_{\text {bol }}\right)_{\text {sat. }}=-3$.

2) Stars with a disk all have the same value of $\log \left(L_{X} / L_{\text {bol }}\right)$ which we will take to be -5.0 .

$H_{\text {disk }} \equiv \log \left(L_{\mathrm{X}} / L_{\text {bol }}\right)_{\text {disk }}=-5$.

Although this value is quite arbitrary, it is compatible with the lowest values of $\log \left(L_{\mathrm{X}} / L_{\mathrm{bol}}\right)$ of low mass $\left(<3 M_{\odot}\right)$ ONC and $\rho$ Ophiuchi stars. According to maximum likelihood distributions, only $\sim 20 \%$ of both the high accretion ONC stars (as defined by Flaccomio et al. 2003b) and of all $\rho$ Ophiuchi members in our sample, have $\log \left(L_{\mathrm{X}} / L_{\text {bol }}\right)$ below this threshold. Note also that in a more realistic model which allows for a continuous range of $L_{\mathrm{X}} / L_{\mathrm{bol}}$ values and of disk evolutionary stages, $H_{\text {disk }}$ would correspond to $\log \left(L_{\mathrm{X}} / L_{\mathrm{bol}}\right)$ of stars with the least evolved accretion disks.

3) The dissipation of circumstellar disks can be described by a stochastic process with characteristic time-scale $\tau_{\text {disk }}$; we can express the fraction of stars that retain their disk, $f_{\text {disk }}$, at a time $t$ by:

$f_{\text {disk }}=\frac{N_{\text {disk }}}{N_{\text {tot }}}=\mathrm{e}^{-t / \tau_{\text {disk }}}$.
We can then compute the mean $\log \left(L_{\mathrm{X}} / L_{\mathrm{bol}}\right)$ as a function of $t$, $<H(t)>$.

$<H(t)>=H_{\text {sat. }}+\left(H_{\text {disk }}-H_{\text {sat. }}\right) \mathrm{e}^{-t / \tau_{\text {disk }}}$.

This model has two unknown parameters: $H_{\text {disk }}$, which we have fixed at -5 , and $\tau_{\text {disk }}$ which we keep as a free parameter. $H_{\text {sat }}$ is here considered as fixed by the observations at -3 , roughly corresponding to the highest values observed in the PMS and equal to the saturation level obtained for fast rotating MS stars. The right column of Fig. 8 shows, superposed on the already described observed trends of $\log \left(L_{\mathrm{X}} / L_{\mathrm{bol}}\right)$ with age for different mass ranges, three realization of the model corresponding to three values of the disk dissipation time-scale, 1.0, 1.5 and 2.0 Myrs. We note two facts:

1) The agreement between data for the PMS regions and models is qualitatively good, also considering the extreme simplicity of our assumptions. The models do not, and are not supposed to, reproduce the fall in $L_{\mathrm{X}} / L_{\mathrm{bol}}$ on the MS, which can be attributed either to wind-driven spin down of the stars or, for the 2-3 $M_{\odot}$ bin, to the loss of the convective region.

2) The observed evolution of $L_{X} / L_{\text {bol }}$ is somewhat faster for more massive stars. The model seems to suggest that $\tau_{\text {disk }}$ depends on stellar mass, ranging from $\sim 2.0$ Myrs for $M \sim 0.2 M_{\odot}$ to $\sim 1.0 \mathrm{Myrs}$ for $M \sim 0.75 M_{\odot}$, where the quoted masses are simply the centers of the second and fourth mass bins.

We therefore seem to have derived an indication on the circumstellar disk lifetime. How do these results compare with totally independent results obtained from specific studies performed at infrared wavelengths? Haisch et al. (2001a) analyze a sample of six clusters and determine, from a linear fit of disk fraction vs. cluster age, a disk lifetime of about $6 \mathrm{Myr}$, this being the age at which essentially all the stars loose their near-IR detectable disks. A similar analysis performed by Hillenbrand (2003) using a larger sample of star forming regions, suggests instead an inner disk lifetime of only 2-3 Myr. An independent indication on the timescale for the evolution of inner disks has been recently derived by Herbst et al. (2002) from the rotational periods of ONC members. Interpreting the period distribution in the context of the disk locking hypothesis, they conclude that the timescale for ONC stars to be released from their disk locking is $0.5-1.0$ times the ONC age. If the two median stellar ages reported in Fig. 1 for our ONC sub-regions are used this translates to $0.7-2.0 \mathrm{Myr}$.

Our best guess for $\tau_{\text {disk }}$ derived from X-ray data, i.e. 12 Myrs, falls within the range of the above estimates. We note that all these estimates are on one hand subject to significant uncertainties, and on the other hand may trace different, though related phenomena such as the presence of the inner disk, disk locking and mass accretion. The order of magnitude agreement is therefore suggestive. Although we have identified the evolutionary time-scale of $L_{\mathrm{X}} / L_{\mathrm{bol}}$ with the disk dissipation timescale, our model is actually rather general: it only assumes a two level population and a fixed transition probability per unit time from the low to the high level. We have tentatively identified the low level with the presence of disks because recent studies (Flaccomio et al. 2003b,c) indicate that stars surrounded by accretion disks have lower levels of $\log \left(L_{\mathrm{X}} / L_{\mathrm{bol}}\right)$. If the lowered activity level is in fact due to accretion itself as 
opposed to the presence of a disk, the time-scale for the evolution of activity might correspond to the time-scale for the evolution of accretion, which might be shorter than the time-scale for the disappearance of IR detectable disks. The rather good agreement of our X-ray derived time scale with the disk locking times scale reported by Herbst et al. (2002) is particularly suggestive as disk locking implies a modification of the magnetic field geometry, conceivably affecting the X-ray emitting plasma.

As for our second result, i.e. the dependence of the X-ray derived $\tau_{\text {disk }}$ from stellar mass, we note that this dependence is also suggested, and in the same direction found here, by several IR studies (e.g. Haisch et al. 2001b; Hillenbrand et al. 1998), thus giving further support to our interpretation.

More X-ray and optical/IR data are needed to fully explore the relation between magnetic activity and accretion and/or disks.

\section{Summary and conclusions}

We have studied the evolution of X-ray activity in the first stages of stellar evolution, from the early PMS ( $\sim 1$ Myr.) to the ZAMS ( $100 \mathrm{Myr})$, using published and archival data on five star forming regions and two young ZAMS clusters. In all cases we have reviewed the X-ray and optical data used to characterize members of the respective regions. If necessary, we have repeated the data analysis in order to homogenize and update, to the best of our knowledge, the assumptions used to derive activity indicators $\left(L_{\mathrm{X}}\right.$ and $\left.L_{\mathrm{X}} / L_{\mathrm{bol}}\right)$ and stellar parameters (masses and ages).

In the early PMS phase we observe that low mass stars that posses a convective region show high levels of activity, often close to the saturation level $\left(\log L_{\mathrm{X}} / L_{\mathrm{bol}} \sim-3\right)$ found for fast rotating MS stars. We compare these activity levels with those predicted by two physically distinct scenarios:

1) the total energy output of the corona $\left(L_{X}\right)$ is determined exclusively by stellar rotation $\left(P_{\text {rot }}\right)$ and saturates when $L_{\mathrm{X}}$ reaches $10^{-3}$ times the total stellar luminosity.

2) the fraction of energy output by the corona respect to the whole energy produced by the star is determined by the dimensionless ratio between the characteristic time of rotation and the characteristic time of convection (i.e. the Rossby number, $R_{0}$ ). This relation saturates at a fixed value of $R_{0}$, corresponding to $L_{\mathrm{X}} / L_{\mathrm{bol}} \sim 10^{-3}$.

While, for MS stars, these two scenarios are hardly distinguishable, both giving a fair description of the observations, they predict distinctively different results if applied to 1-5 Myr old stars. Scenario 1, in order to explain the high levels of activity observed, would require very fast rotations, which are simply not observed. Scenario 2 on the other hand predicts that stars in the early PMS with $P_{\text {rot }} \lesssim 16$ d, i.e. consistent with the rotations measured in the $\mathrm{ONC}$, should all be saturated.

Our data seem therefore to favor scenario 2, although it does not fully account for the observations: median $L_{\mathrm{X}} / L_{\mathrm{bol}}$ appears to be somewhat lower than the saturation value for young ages $(\lesssim 5 \mathrm{Myr})$ and low masses $\left(\lesssim 1 M_{\odot}\right)$, but tends to reach that level at older ages and larger masses. Saturation is then retained up to the age of the Pleiades and NGC 2516
(100-140 Myrs) for stars with $M \lesssim 0.5 M_{\odot}$, while activity in more massive stars seems to desaturate earlier. We can tentatively explain this latter decay of activity from the PMS to the MS with the decrease of the convective turnover time (or, equivalently, convective zone depth), which is indeed more pronounced for more massive stars (Ventura et al. 1998) and, possibly, with the angular momentum loss induced by magnetic winds (cf. Bouvier et al. 1997). The increase of $L_{X} / L_{\mathrm{bol}}$ in the early PMS however is not so readily explained by the foreseen evolution of the Rossby number. Supported by observational evidence, we put forth a simple model that assumes the existence of two populations: one in which activity is at the saturation level, the other with lower observed activity, which we tentatively identify with stars surrounded by circumstellar disks and/or undergoing accretion. We then postulate that the low activity population makes a transition to the high activity population (e.g. dissipates disks or stops accreting) with a characteristics time, $\tau$. Observed median activity levels are roughly consistent with such a model if $\tau \sim 1-2 \mathrm{Myr}$, being at the short end of the interval for higher mass stars $\left(\sim 1 M_{\odot}\right)$ and at the long end for the lowest mass ones $\left(\sim 0.2 M_{\odot}\right)$. These characteristic times are similar to disk dissipation times derived by IR studies, to the timescale for disk locking, as derived by modeling the rotational periods distribution of ONC stars, and to the expected timescale for the evolution of circumstellar accretion. Given the considerable uncertainties in all of these estimates, we restrain from any more precise identification of the physical process that determines the evolution of activity. We also note that the observed trend of $\tau$ with mass is consistent, according to recent IR studies, with the disk (and possibly with the accretion and disk locking) hypothesis.

Acknowledgements. The authors are thankful to P. Ventura for providing his model calculation of convective turnover times in the PMS and to the referee, T. Preibisch, for comments that helped improve this work. E.F. wish to thank F. Damiani for providing the X-ray data for NGC 2516 prior to publication and N. Pizzolato for useful discussions. The authors wish to acknowledge support from the Italian Space Agency (ASI) and MURST.

\section{References}

Bouvier, J., Forestini, M., \& Allain, S. 1997, A\&A, 326, 1023

Dame, T. M., Ungerechts, H. Cohen, R. S., et al. 1987, ApJ, 322, 706

Damiani, F., Maggio, A., Micela, G., \& Sciortino, S. 1997, ApJ, 483, 350

Damiani, F., \& Micela, G. 1995, ApJ, 446, 341

Damiani, F., Flaccomio, E., Micela, G., et al. 2003, ApJ, in press

Daniel, K. J., Linsky, J. L., \& Gagné, M. 2002 ApJ, 578, 486

de Zeeuw, P. T., Hoogerwerf, R., de Bruijne, J. H. J., Brown, A. G. A., \& Blaauw, A. 1999, AJ, 117, 354

Feigelson, E. D., \& Nelson, P. I. 1985, ApJ, 293, 192

Feigelson, E. D., Casanova, S., Montmerle, T., \& Guibert, J. 1993, ApJ, 416, 623

Feigelson, E. D., Broos, P., Gaffney, J. A., et al. 2002, ApJ, 574, 258

Feigelson, E. D., Gaffney, J. A., Garmire, G., Hillenbrand, L. A., \& Townsley, L. 2003, ApJ, 584, 911

Flaccomio, E., Micela, G., Sciortino, S., et al. 2000, A\&A, 355, 651 
Flaccomio, E., Damiani, F., Micela, G., et al. 2003, ApJ, 582, 382

Flaccomio, E., Damiani, F., Micela, G., et al. 2003, ApJ, 582, 398

Flaccomio, E., Micela, G., \& Sciortino, S. 2003, A\&A, 397, 611

Flaccomio, E. 2002, Ph.D. Thesis, University of Palermo

Gagne, M., Caillault, J., \& Stauffer, J. R. 1995, ApJ, 450, 217

Haisch, K. E., Lada, E. A., \& Lada, C. J. 2001a, ApJ, 553, L153

Haisch, K. E., Lada, E. A., \& Lada, C. J. 2001b, AJ, 121, 2065

Hartmann, L. 2001, AJ, 121, 1030

Hartmann, L. 2002, ApJ, 566, L29

Herbst, W., Bailer-Jones, C. A. L., Mundt, R., Meisenheimer, K., \& Wackermann, R. 2002, A\&A, 396, 513

Hillenbrand, L. A. 1997, AJ, 113, 1733

Hillenbrand, L. A., Strom, S. E., Calvet, N., et al. 1998, AJ, 116, 1816

Hillenbrand, L. A. 2003, Origins 2002: The Heavy Element Trail from Galaxies to Habitable Worlds, ed. C. E. Woodward, \& E. P. Smith, ASP Conf. Ser.

Imanishi, K., Koyama, K., \& Tsuboi, Y. 2001, ApJ, 557, 747

Imanishi, K., Tsujimoto, M., \& Koyama, K. 2001, ApJ, 563, 361

Jeffries, R. D., Thurston, M. R., \& Pye, HJ. P. 1997, MNRAS, 287, 350

Jeffries, R. D., Thurston, M. R., \& Hambly, N. C. 2001, A\&A, 375, 863

Kenyon, S. J., \& Hartmann, L. 1995, ApJS, 101, 117

Kenyon, S. J., Lada, E. A., \& Barsony, M. 1998, AJ, 115, 252

Lawson, W. A., Feigelson, E. D., \& Huenemoerder, D. P. 1996, MNRAS, 280, 1071

Lawson, W. A., Crause, L. A., Mamajek, E. E., \& Feigelson, E. D. 2001, MNRAS, 321, 57

Lawson, W. A., Crause, L. A., Mamajek, E. E., \& Feigelson, E. D. 2002, MNRAS, 329, L29

Luhman, K. L., \& Rieke, G. H. 1999, ApJ, 525, 440

Mamajek, E. E., Lawson, W. A., \& Feigelson, E. D. 1999, ApJ, 516, L77

Mamajek, E. E., Lawson, W. A., \& Feigelson, E. D. 2000, ApJ, 544, 356

Mathis, J. S. 1990, ARA\&A, 28, 37

Meynet, G., Mermilliod, J.-C., \& Maeder, A. 1993, A\&AS, 98, 477
Micela, G., Sciortino, S., Kashyap, V., Harnden, F. R., \& Rosner, R. 1996, ApJS, 102, 75

Micela, G., Sciortino, S., Harnden, F. R., \& Rosner, R. 1998, Ap\&SS, 261, 105

Micela, G., Sciortino, S., Harnden, F. R. Jr., et al. 1999, A\&A, 341, 751

Montmerle, T., Grosso, N., Tsuboi, Y., \& Koyama, K. 2000, ApJ, 532, 1097

Muench, A. A., Alves, J., Lada, C. J., \& Lada, E. A. 2001, ApJ, 558, L51

Neuhäuser, R., Sterzik, M. F., Schmitt, J. H. M. M., Wichmann, R., \& Krautter, J. 1995, A\&A, 297, 391

Noyes, R. W., Hartmann, L. W., Baliunas, S. L., Duncan, D. K., \& Vaughan, A. H. 1984, ApJ, 279, 763

Pallavicini, R., Golub, L., Rosner, R., et al. 1981, ApJ, 248, 279

Peres, G., Orlando, S., Reale, F., Rosner, R., \& Hudson, H. 2000, ApJ, 528, 537

Pizzolato, N., Maggio, A., Micela, G., Sciortino, S., \& Ventura, P. 2003, A\&A, 397, 147

Predehl, P., \& Schmitt, J. H. M. M. 1995, A\&A, 293, 889

Preibisch, T., \& Zinnecker, H. 2002, AJ, 123, 1613

Randich, S. 2000, Stellar Clusters and Associations: Convection, Rotation, and Dynamos, ed. R. Pallavicini, G. Micela, \& S. Sciortino, ASP Conf. Ser., 198, 401

Rebull, L. M., Wolff, S. C., Strom, S. E., \& Makidon, R. B. 2002, AJ, 124,546

Rebull, L. M., Makidon, R. B., Strom, S. E., et al. 2002, AJ, 123, 1528

Ryter, CH. E. 1996, Ap\&SS, 236, 285

Siess, L., Dufour, E., \& Forestini, M. 2000, A\&A, 358, 593

Stauffer, J. R., Caillault, J.-P., Gagne, M., Prosser, C. F., \& Hartmann, L. W. 1994, ApJS, 91, 625

Stelzer, B., \& Neuhäuser, R. 2001, A\&A, 377, 538

Vaiana, G. S., Fabbiano, G., Giacconi, R., et al. 1981, ApJ, 245, 163

Ventura, P., Zeppieri, A., Mazzitelli, I., \& D'Antona, F. 1998, A\&A, 334, 953

Vrba, F. J., Coyne, G. V., \& Tapia, S. 1993, AJ, 105, 1010 